\title{
Experimental study of bidirectional seiching in an open-channel, lateral cavity in the time and frequency domain
}

\author{
L. Engelen $\odot,{ }^{1}$ C. Perrot-Minot $\odot,{ }^{2}$ E. Mignot $\odot,{ }^{2}$ N. Rivière $\odot,{ }^{2}$ and T. De Mulder $\odot{ }^{1}$ \\ ${ }^{1}$ Hydraulics Laboratory, Department of Civil Engineering, Ghent University, 9000 Ghent, Belgium \\ ${ }^{2}$ Université de Lyon, INSA Lyon, Ecole Centrale de Lyon, Université Claude Bernard Lyon I, CNRS, LMFA, \\ UMR 5509, F-69621 Villeurbanne, France
}

(Received 24 January 2020; accepted 27 July 2020; published 1 October 2020)

\begin{abstract}
This experimental laboratory study presents a detailed analysis of the surface oscillations inside a square cavity connected laterally to an open channel. For an approaching steady and one-dimensional main stream with a high (subcritical) Froude number, interaction with the dead water volume in the cavity area triggers high-amplitude free-surface oscillations, denoted seiches. In the present study, the length of the square cavity equals half of the combined width of the cavity and the main channel. This particular geometry enables two-dimensional or bidirectional seiching with standing waves being excited in both directions simultaneously. In this work, the (relative) magnitude of the different seiching modes (transverse and longitudinal) is measured and analyzed for a range of flow conditions with varying Froude number. The current experimental results confirm earlier findings indicating that the magnitude and dominance of the longitudinal seiching mode (with respect to the transverse mode) grows with increasing Froude number of the main stream. Additionally, a wavelet analysis in the time and frequency domains reveals that the amplitude of the two modes fluctuates significantly in the course of a single experiment, such that seiching cannot be considered a steady phenomenon. Therefore, two alternative strategies are presented to quantify the amplitude of both modes using a more time-localized approach that is less affected by the intermittent behavior of seiching. Although more research is necessary to fully understand what determines the dominant mode at a given moment, an in-depth analysis of the surface recordings at multiple locations in the cavity suggests that the transition from transverse to longitudinal seiching (and vice versa) is related to the phase difference between the bidirectional seiching waves at the (inner) corners of the cavity.
\end{abstract}

DOI: 10.1103/PhysRevFluids.5.104801

\section{INTRODUCTION}

Open-channel lateral cavities are semienclosed embayments adjacent to a main stream (e.g., a fluvial or coastal current) flowing past their open end. Reference [1] mentioned that such lateral embayments can be subdivided into three main categories, each characterized by a different influence of cavities located upstream and downstream with respect to the studied embayment: (a) isolated cavities (as in the present case), (b) a series of lateral cavities with a chosen spacing and in one or both of the main channel sidewalls (e.g., studied in Refs. [2-4]), and (c) cavities located between two consecutive groynes (e.g., studied in Refs. [5-8]). For category (a), no other cavities influence the approaching flow in the main channel, such that the upstream part of the main stream is directed parallel to the side walls of the main channel. For category (b), the mean upstream flow is still directed parallel to the side walls, but the flow is affected by turbulent structures generated in the mixing layer of the upstream dead zone. Finally, for category (c), the 
transverse component of the main stream upstream of the cavity is directed toward the center of the main stream. These significant differences influence the flow characteristics in and around these embayments and significantly influence the mass and momentum exchange between the cavities and the main stream. The present paper is dedicated to isolated lateral cavities [category (a)], which can be formed naturally, e.g., oxbows, cut-off meanders, and natural irregularities of the banks, or by human interventions, e.g., turning basins in waterways, harbors, docks, marinas, and entrances to locks.

The recirculating flow within the cavity exchanges mass and momentum with the main stream through the mixing layer that develops at the interface between the main stream and the cavity [9-11]. This mixing layer is characterized by the advection of quasiperiodic, large-scale vortices $[10,12,13]$ that are shed from the upstream corner of the interface. Their impingement $[3,14]$ on the downstream corner of the interface excites local free-surface oscillations and an intermittent inflow toward the cavity $[6,9]$.

Within such a lateral cavity, free-surface oscillations with important amplitude may be generated by various (hydrodynamic or meteorological) excitation mechanisms. In case of a harbor dock, e.g., such surface conditions may be detrimental for cargo handling of ships present inside the basin. This paper is devoted to one of the less-known sources of surface oscillations, denoted seiching, in which large standing gravity waves within the cavity are excited by the interaction of the cavity flow with the main stream for certain combinations of cavity geometry and main channel flow conditions. Besides influencing the surface conditions and flow field within the cavity, the results of Refs. $[12,15,16]$ indicated (even if not proven directly) that seiching increases the mass exchange coefficient up to $40 \%$ compared to cases without seiching. Reference [12] suggested that the impact of seiching on the mass exchange is related to the increased turbulent kinetic energy and Reynolds stresses in the interface region. Additionally, Ref. [4] showed that seiching has a significant impact on the in-cavity sedimentation processes and the total amount of sediment trapped within the cavity. Finally, Ref. [15] suggested that transverse gravity standing waves (see further) increase the mass exchange coefficient more significantly compared to longitudinal standing waves. While not exactly similar to the present cavity geometry, experimentally measured values of the (velocity-based) mass exchange coefficient in two sequential cavities indicated a 50-74\% increase during transverse seiching, relative to longitudinal seiching.

Lateral embayments considered in laboratory studies are often simplified by a square or a rectangular planform geometry at right angles with the main stream, which makes it possible to analytically compute the natural frequencies of the basin. Previous research, e.g., Refs. [12,17,18], suggested that seiching is triggered in case the natural frequency of the cavity is in agreement with the passing frequency of the vortices in the mixing layer, while Refs. [12,16] found that also the flapping or jitter phenomenon of the mixing layer behaves in phase with the free-surface oscillations. Reference [3] also found an agreement between the surface oscillations and the periodic impingement of mixing layer's vortices on the downstream corner of the cavity and suggested the existence of critical values of the Strouhal number for which surface oscillations are maximally excited. Nonetheless, it remains difficult to predict the oscillation direction and the number of nodes of the standing waves.

Reference [19] showed that for a fixed rectangular cavity geometry, the selected mode of seiching is mostly governed by the Froude number of the main stream. In this respect, Refs. [12,20] showed that two different flow regimes exist depending on the Froude number. For a low Froude number, i.e., typically $\mathrm{Fr} \lesssim 0.60$, [20] refer to the pre-locked-on state where they observed oscillations with a limited amplitude and with a frequency increasing linearly with Fr. This linear increase was explained in Ref. [21] using a so-called feedback model based on the traveling times along the mixing layer of both vortices (toward downstream) and free-surface gravity waves (toward upstream). In contrast, for $\mathrm{Fr}>0.60$, Refs. [12,18,20,22] suggested a locked-on regime in which the frequency of the coupled oscillations remained fixed at, or locked-on, the natural frequency of the cavity over a range of Froude numbers. In this high-Froude-number regime, Refs. [12,20] observed the oscillation amplitude to increase with increasing Froude number up to $\mathrm{Fr} \approx 0.7$, followed by 
a decreasing amplitude for higher Froude numbers. Reference [23] observed for a constant Froude number but with a varying width of the cavity that a transition from longitudinal to transverse seiching occurs as the width to length ratio of the rectangular cavity exceeds 2 .

However, most authors only reported a single mode of seiching in either the transverse or longitudinal direction, thus assuming a unidirectional sinusoidal surface shape. In contrast, for a rectangular cavity, Reference [19] proved that multiple seiching modes in a single oscillation direction can be excited simultaneously. For a square cavity with a side length equal to the width of the main stream, Ref. [18] observed that waves in both the longitudinal and transverse direction can actually take place simultaneously. Reference [22] investigated the relative magnitude of transverse and longitudinal seiching in function of the Froude number of the main stream, in which they found a transitional Froude number for which the dominant seiching mode (i.e., having the largest amplitude) changed from transverse to longitudinal seiching.

In this paper, only the free-surface characteristics (oscillation frequency, amplitude and shape) during seiching will be discussed. The primary focus of this work will be the large-scale standing waves, while local, small surface perturbations will not be discussed in detail. While seiching also influences the flow in and around the cavity and is expected to enhance mass and momentum exchange between the main stream and the cavity, an analysis of the flow field below the standing waves will not be explored in the present contribution. This work is particularly dedicated to the investigation of a cavity with specific geometrical constraints, i.e., where the width of the main channel and the width and length of the cavity are all three equal to each other (thus a square cavity). For such a particular geometry, bidirectional seiching is assumed to be promoted by the fact that some natural frequencies of transverse and longitudinal seiching are then equal or a multiple of each other. Moreover, for this fixed geometry, seiching is expected to depend on three parameters (that will be defined in Sec. III A): the Froude number (Fr), the dimensionless water depth $\left(h_{m} / L\right)$, and the Reynolds number (Re) of the main stream. For Re sufficiently high and a fixed $h_{m} / L$, only Fr is expected to affect the excited seiching mode(s).

The first objective of this work is to refine previous results reported in Ref. [22] and investigate the relative magnitude of the different seiching modes (longitudinal and transverse) for a range of flow conditions with varying Froude number. Based on the registrations of the free surface in a series of laboratory experiments, the characteristic frequencies and corresponding amplitudes of the standing waves are first determined using a Fourier analysis of the measured recordings. As a second objective, this study investigates the unsteady character of the bidirectional seiching phenomenon, in which it will be shown that the dominant mode of seiching alternates during the course of a single experiment. For that purpose, a wavelet analysis [24] will be adopted to investigate the time-varying magnitude of the seiching waves in both the time and frequency domains. Finally, a theoretical estimation of the free-surface oscillations will be presented based on a phase criterion at the inner corners of the cavity. Subsequently, this will be compared with the experimental free-surface registrations in the respective corners of the cavity to identify possible situations for mode switching to occur.

\section{THEORETICAL BACKGROUND OF SEICHING}

In a (semi-)enclosed basin, long-period standing surface oscillations may occur in case the forcing frequency equals a natural frequency of the basin. When such standing gravity waves occur in (relatively shallow) harbors and lakes, they are often called seiches [25]. Laboratory experiments [12,17-20,23] on seiches mainly investigate basins with a simplified planform geometry, like, e.g., a rectangular or, as in the present case, square shape. The cavities are usually connected at right angles with a main stream characterized by a steady one-dimensional (1D) flow and have most often sharp edges, smooth walls, a flat bed, and no step between the main stream and the cavity. The peak frequencies of the free-surface oscillations inside such basins have been observed to approximately match the natural frequencies of closed rectangular (or square) basins with the same dimensions $[25,26]$. 
In general, for a water body in a closed rectangular basin with a mean water depth $h_{m}$ and horizontal dimensions $L_{l}$ (longitudinal direction, denoted $x$ ) and $L_{t}$ (transverse direction, denoted $y$ ), it is possible to mathematically describe the free-surface oscillations in time. In the following, the variable free-surface elevation will be expressed relative to the mean water depth $h_{m}$, i.e.,

$$
\eta(x, y, t)=h(x, y, t)-h_{m},
$$

in which $\eta(x, y, t)$ denotes the instantaneous position of the free surface with respect to $h_{m}$ at horizontal position $(x, y)$ and time instance $t$, and $h(x, y, t)$ denotes the corresponding instantaneous surface elevation above the bed (i.e., the local water depth). For standing waves with a frequency $f_{n_{l} n_{t}}$, the mathematical description of $\eta(x, y, t)$ is given by [27]

$$
\begin{aligned}
\eta(x, y, t) & =h(x, y, t)-h_{m} \\
& =\sum_{n_{l}, n_{t}} \cos \left(n_{l} \frac{x \pi}{L_{l}}\right) \cos \left(n_{t} \frac{y \pi}{L_{t}}\right) \exp \left(2 \pi i f_{n_{l} n_{t}} t\right),
\end{aligned}
$$

in which $i$ denotes the imaginary unit $\left(i^{2}=-1\right)$, and $n_{l}$ and $n_{t}$ indicate the number of nodes of the sinusoidal surface shape in the longitudinal and transverse direction, respectively, such that both $n_{l}$ and $n_{t} \geqslant 0$ and $n_{l}+n_{t} \neq 0$. In the particular case of one-dimensional seiching in either one of both directions, distinction is made between transverse seiching $\left(n_{l}=0\right.$ and $\left.n_{t}>0\right)$ and longitudinal seiching $\left(n_{l}>0\right.$ and $\left.n_{t}=0\right)$. The time-dependent behavior of the standing waves is characterized by the frequency $f_{n_{l} n_{t}}$, which can theoretically $[25,27]$ be predicted by

$$
f_{n_{l} n_{t}}=\frac{1}{2 \pi} \sqrt{\pi g \sqrt{\left(\frac{n_{l}}{L_{l}}\right)^{2}+\left(\frac{n_{t}}{L_{t}}\right)^{2}} \tanh \left[\pi h_{m} \sqrt{\left(\frac{n_{l}}{L_{l}}\right)^{2}+\left(\frac{n_{t}}{L_{t}}\right)^{2}}\right]},
$$

with $g$ the gravitational acceleration. In this work, the different seiching modes will be distinguished based on the number of nodes of the corresponding surface deformation in both directions. Following Eq. (2), the seiching mode with $n_{l}$ nodes in the longitudinal direction (along the $x$ axis) and $n_{t}$ nodes in the transverse direction (along the $y$ axis) will be denoted by $m_{n_{l} n_{t}}$.

Previous experiments on flow past a lateral cavity $[18,22]$ showed that bidirectional seiching may occur in the specific case where the streamwise length scale $L_{l}=L$ equals half of the crosswise length scale $L_{t}=b+W$, in which $b$ is the width (along the $y$ axis) of the main channel and $L$ and $W$ are the length (along the $x$ axis) and width (along the $y$ axis) of the cavity, respectively [see Fig. 1(b)]. Substitution of $L_{t}=2 L_{l}$ in Eq. (3) implies that the natural frequencies of unidirectional transverse seiching $\left(n_{l}=0, n_{t} \neq 0\right)$ equal half of the unidirectional longitudinal frequencies $\left(n_{l} \neq 0, n_{t}=0\right)$. As a consequence, the pair modes of transverse seiching with frequencies $f_{02}, f_{04}, f_{06}, \ldots$, have (theoretically) the same frequency as the longitudinal modes with frequencies $f_{10}, f_{20}, f_{30}, \ldots$, (a situation referred to as degeneracy in Refs. [26,28]).

\section{EXPERIMENTAL METHODOLOGY}

\section{A. Laboratory facility}

The experiments described in this work are conducted in the Hydraulics Laboratory of Ghent University, Belgium. The cavity facility (Fig. 1) consists of a polycarbonate-walled flume (Manning coefficient of polycarbonate $\approx 0.01 \mathrm{~s} \mathrm{~m}^{-1 / 3}$ ) with a total length of $12 \mathrm{~m}$ and a rectangular cross section with a width $b=0.40 \mathrm{~m}$. The main channel is connected to a square side embayment, having a length $L$ and width $W$ both equal to $0.40 \mathrm{~m}$ (thus $L=W=b$ ), with the upstream corner of the cavity located at $3 \mathrm{~m}$ downstream from the main channel inlet.

While the cavity has a horizontal bed at the same level as the adjacent main channel zone, the main channel has a bottom slope downstream with respect to the cavity of approximately $1 \%$ to reach higher flow velocities with a limited water depth, i.e., higher (subcritical) Froude numbers in 

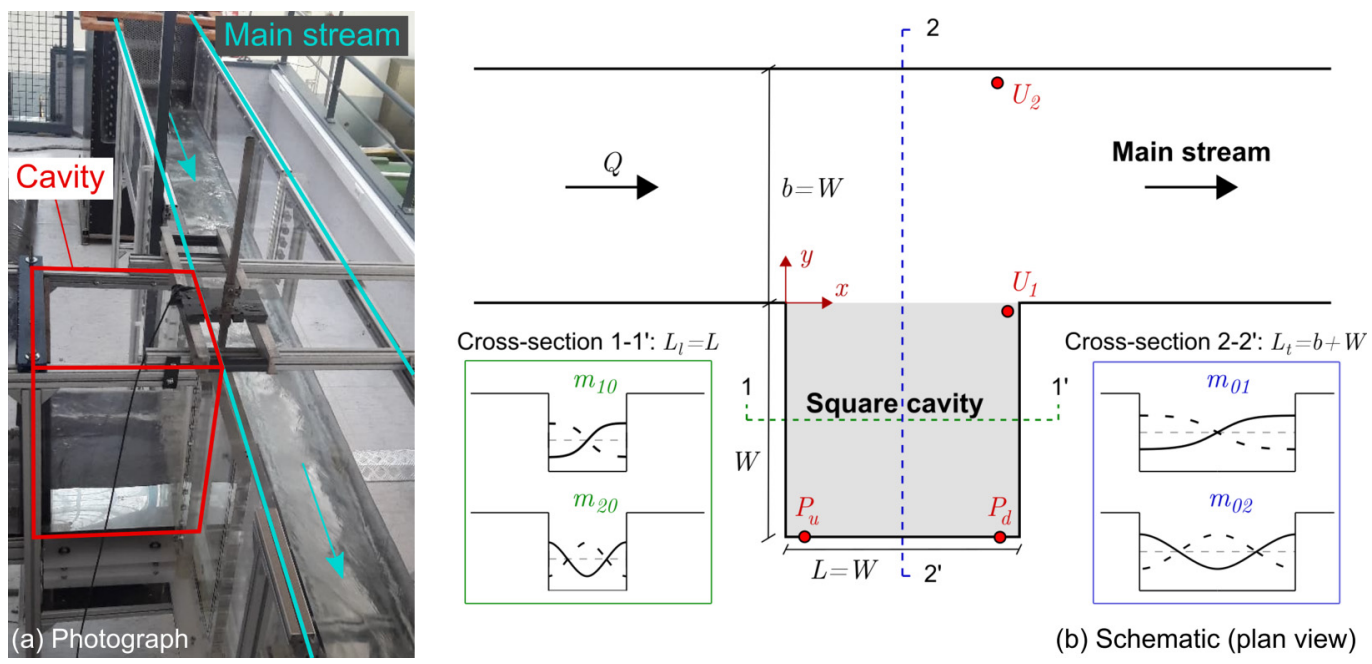

(b) Schematic (plan view)

FIG. 1. Photograph (a) and schematic (b) of the experimental set-up, indicating the location of the upstream $\left(P_{u}\right)$ and downstream $\left(P_{d}\right)$ pressure sensors in the water retaining wall at the closed end of the cavity, as well as the measurement positions of the ultrasonic gauge $\left(U_{1}\right.$ and $\left.U_{2}\right)$. The first two theoretical seiching modes in both the longitudinal $x$ direction $\left(m_{10}, m_{20}\right)$ and transverse $y$ direction $\left(m_{01}, m_{02}\right)$ are sketched for respectively cross section 1-1' (with length scale $L_{l}=L$ ) and cross section 2-2' (with length scale $L_{t}=b+W$ ) for the time instances at which they reach their maximum amplitude.

the main stream in front of the cavity. Multiple honeycomb screens are installed at the upstream boundary condition (main channel inlet) to promote a fully developed flow, whereas an automated weir system is positioned at the downstream boundary condition to control the water level in the flume. The water depth $h_{m}$ is defined as the mean of the time-averaged water depths of the two pressure sensors that are located in the cavity area (see further). For the current experiments, $h_{m}$ is kept constant for all flow cases and equals $14.5 \mathrm{~cm}$, thus $h_{m} / L \simeq 0.36$, which is relatively large to maximize the range of Froude numbers available during the experiments. The main stream discharge $Q$ is measured within the pumping loop using an electromagnetic flow meter, which corresponds to a bulk velocity of the main stream $U_{b}=Q / b h_{m}$. The Reynolds and Froude numbers of the main stream are defined as $\operatorname{Re}=\left(U_{b} D_{h}\right) / v$ and $\mathrm{Fr}=U_{b} / \sqrt{g h_{m}}$, respectively, in which $D_{h}=4 b h_{m} /(b+$ $2 h_{m}$ ) denotes the hydraulic diameter of the main channel, $v$ is the kinematic viscosity of water $\left(1.003 \times 10^{-6} \mathrm{~m}^{2} \mathrm{~s}^{-1}\right.$ for water at $\left.20^{\circ} \mathrm{C}\right)$, and $g$ is the gravitational acceleration. Table I gives a complete overview of the flow cases discussed in this work, in which the 33 flow configurations are ordered by increasing Froude number. The last two rows of Table I correspond to two additional tests with a different measurement position of the ultrasonic gauge compared to the other 33 tests (see further).

The flow conditions are chosen to cover a large range of Froude numbers, while ensuring a sufficiently high Reynolds number such that viscosity effects are considered to be of minor importance. The lowest Froude number $(\mathrm{Fr}=0.3)$ is significantly smaller than the Froude numbers with significant seiching in the literature, i.e., typically $\mathrm{Fr} \gtrsim 0.60[3,12,17-20,23]$. Therefore, flow configurations with low Froude numbers studied in this work correspond to the so-called pre-locked-on regime [20], i.e., with no high-amplitude free-surface oscillations. To investigate the influence of Fr on the (bidirectional) seiching phenomenon, the Froude number is gradually increased in the different tests up to $\mathrm{Fr}=0.68$. The latter is the highest Froude number that could be achieved for the chosen, fixed water depth and is similar to the Froude numbers for which [12,20] observed the oscillation amplitude to attain its maximum value. 
TABLE I. Overview of the studied flow configurations. The first 33 tests are performed with the ultrasonic gauge positioned at $U_{1}$, i.e., near the downstream cavity corner just next to the main stream. Two additional tests (last two rows) are done with the ultrasonic gauge at $U_{2}$, i.e., at the main channel wall opposite to the cavity.

\begin{tabular}{|c|c|c|c|c|c|}
\hline$Q\left(1 \mathrm{~s}^{-1}\right)$ & $h_{m}(\mathrm{~m})$ & $U_{b}\left(\mathrm{~m} \mathrm{~s}^{-1}\right)$ & $h / L$ & $\mathrm{Re}$ & $\mathrm{Fr}$ \\
\hline 21.21 & 0.147 & 0.36 & 0.37 & $1.22 \times 10^{5}$ & 0.30 \\
\hline 24.24 & 0.146 & 0.41 & 0.37 & $1.40 \times 10^{5}$ & 0.35 \\
\hline 35.09 & 0.147 & 0.60 & 0.37 & $2.02 \times 10^{5}$ & 0.50 \\
\hline 38.18 & 0.146 & 0.65 & 0.37 & $2.20 \times 10^{5}$ & 0.55 \\
\hline 39.28 & 0.146 & 0.67 & 0.36 & $2.27 \times 10^{5}$ & 0.56 \\
\hline 39.94 & 0.145 & 0.69 & 0.36 & $2.31 \times 10^{5}$ & 0.58 \\
\hline 40.38 & 0.146 & 0.69 & 0.36 & $2.33 \times 10^{5}$ & 0.58 \\
\hline 40.73 & 0.145 & 0.70 & 0.36 & $2.35 \times 10^{5}$ & 0.59 \\
\hline 41.42 & 0.145 & 0.71 & 0.36 & $2.39 \times 10^{5}$ & 0.60 \\
\hline 41.46 & 0.145 & 0.71 & 0.36 & $2.39 \times 10^{5}$ & 0.60 \\
\hline 41.54 & 0.145 & 0.72 & 0.36 & $2.40 \times 10^{5}$ & 0.60 \\
\hline 41.44 & 0.145 & 0.71 & 0.36 & $2.40 \times 10^{5}$ & 0.60 \\
\hline 42.00 & 0.145 & 0.72 & 0.36 & $2.43 \times 10^{5}$ & 0.60 \\
\hline 42.67 & 0.145 & 0.74 & 0.36 & $2.47 \times 10^{5}$ & 0.62 \\
\hline 44.08 & 0.146 & 0.76 & 0.36 & $2.54 \times 10^{5}$ & 0.63 \\
\hline 43.84 & 0.145 & 0.76 & 0.36 & $2.53 \times 10^{5}$ & 0.63 \\
\hline 44.35 & 0.145 & 0.76 & 0.36 & $2.56 \times 10^{5}$ & 0.64 \\
\hline 44.14 & 0.144 & 0.76 & 0.36 & $2.56 \times 10^{5}$ & 0.64 \\
\hline 44.65 & 0.145 & 0.77 & 0.36 & $2.58 \times 10^{5}$ & 0.64 \\
\hline 44.65 & 0.145 & 0.77 & 0.36 & $2.58 \times 10^{5}$ & 0.65 \\
\hline 44.65 & 0.145 & 0.77 & 0.36 & $2.58 \times 10^{5}$ & 0.65 \\
\hline 43.65 & 0.142 & 0.77 & 0.36 & $2.54 \times 10^{5}$ & 0.65 \\
\hline 44.85 & 0.145 & 0.78 & 0.36 & $2.59 \times 10^{5}$ & 0.65 \\
\hline 44.89 & 0.144 & 0.78 & 0.36 & $2.60 \times 10^{5}$ & 0.65 \\
\hline 45.29 & 0.145 & 0.78 & 0.36 & $2.62 \times 10^{5}$ & 0.65 \\
\hline 45.43 & 0.145 & 0.78 & 0.36 & $2.62 \times 10^{5}$ & 0.66 \\
\hline 45.41 & 0.144 & 0.79 & 0.36 & $2.63 \times 10^{5}$ & 0.66 \\
\hline 46.22 & 0.146 & 0.79 & 0.36 & $2.67 \times 10^{5}$ & 0.66 \\
\hline 46.26 & 0.145 & 0.80 & 0.36 & $2.68 \times 10^{5}$ & 0.67 \\
\hline 46.85 & 0.146 & 0.80 & 0.36 & $2.70 \times 10^{5}$ & 0.67 \\
\hline 47.07 & 0.146 & 0.81 & 0.36 & $2.71 \times 10^{5}$ & 0.67 \\
\hline 46.63 & 0.145 & 0.81 & 0.36 & $2.70 \times 10^{5}$ & 0.68 \\
\hline 47.00 & 0.145 & 0.81 & 0.36 & $2.71 \times 10^{5}$ & 0.68 \\
\hline 40.70 & 0.143 & 0.71 & 0.36 & $2.36 \times 10^{5}$ & 0.60 \\
\hline 42.83 & 0.142 & 0.75 & 0.36 & $2.49 \times 10^{5}$ & 0.64 \\
\hline
\end{tabular}

Between two consecutive tests, the cavity is closed off from the main stream to prevent biased results due to influences of previous flow regimes, i.e., prevent from any hysteresis. Subsequently, a sufficient waiting time prior to the start of recording $(10 \mathrm{~min})$ ensures that no initialization effects influence the results.

To determine the time-dependent, three-dimensional shape of the water surface inside the cavity, a combination of three synchronized single-point measurement devices is used. The first device is an ultrasonic gauge (UNDK 20I6912/S35A of Baumer) with an uncertainty of less than $0.5 \mathrm{~mm}$. For the majority of the experiments presented in this paper, it is positioned near the downstream edge of the cavity $\left[U_{1}\right.$ in Fig. 1(b)] to reliably distinguish the dominating frequencies of the impinging mixing layer. Additionally, a limited set of flow cases is repeated with the ultrasonic 
gauge positioned at $U_{2}$ in Fig. 1(b) to confirm that the measured seiching modes correspond with the theoretical sinusoidal surface shapes depicted in Fig. 1(b). In addition to the ultrasonic gauge, two pressure sensors (UNIK5000 of GE Measurement \& Control) register the time evolution of the water depth with a measurement accuracy of $\pm 0.05 \mathrm{~mm}$ at the two inner corners inside the cavity [ $P_{u}$ and $P_{d}$ in Fig. 1(b)]. All three devices are synchronized and measure the surface oscillations with a sampling frequency of $50 \mathrm{~Hz}$ over a total measurement time of $90 \mathrm{~min}$.

For relatively high Froude numbers, the vortices shed at the upstream corner of the cavity significantly disturb the local surface shape during impingement on the cavity corner near $U_{1}$. Therefore, the amplitudes measured by the ultrasonic gauge (at $U_{1}$ ) are considered suboptimal to estimate the amplitude of the standing waves inside the cavity. Similarly, the surface registrations by the downstream pressure sensor $\left(P_{d}\right)$ are influenced by the fluctuating inflow of vortices entering the cavity after impingement [13], creating a local enhancement of the potential energy but also local surface irregularities. In contrast, the upstream pressure sensor $\left(P_{u}\right)$ seems less affected by the vortex dynamics (i.e., the typical free-surface depressions occurring with vortices) and mean recirculating flow within the cavity, which motivates the use of the upstream pressure registrations to investigate the amplitude of seiching in function of the Froude number in Sec. IV. Although not adopted to estimate the amplitude of the seiching waves, the downstream pressure sensor and ultrasonic depth gauge are useful to distinguish the type of seiching mode by evaluating the cross spectra between the three recorded time series. Herewith, the phase difference (not shown in this paper) evaluated at the characteristic seiching frequencies matches the theoretically expected sinusoidal surface shapes, along both the longitudinal and transverse directions.

\section{B. Analysis methodology}

The first objective of this work is to refine the results presented in Ref. [22], in which the influence of the Froude number on the seiching waves was investigated, albeit with a nonconstant water depth. Therefore, a Fourier transform is applied to the surface registration ( $90 \mathrm{~min}$ in total) to compute the power spectral density $\left(\operatorname{Psd}_{\mathrm{FT}}\right)$, i.e., the energy of the local surface deformation as a function of the frequency. Next the amplitude (denoted $\Delta \eta_{\mathrm{FT}}$ in this work) of the different seiching modes is derived by integration of the $\mathrm{Psd}_{\mathrm{FT}}$ of the upstream pressure sensor recordings around the peaks of the spectrum.

However, as will be confirmed by the results presented in this paper, visual analysis of the registered surface oscillations showed that the bidirectional seiching waves vary significantly in amplitude during the total measurement period (lasting $90 \mathrm{~min}$ ). Although the main stream flow is steady, $1 \mathrm{D}$, and constant for a single experiment, this causes the dominant mode of seiching to alternate between the longitudinal and transverse mode. To investigate this intermittent behavior of the seiching phenomenon and determine the time-varying magnitude of the seiching modes, this work also employs a wavelet analysis [24]. In analogy with the Fourier transform (FT), which decomposes a signal in a series of sine waves with different frequencies, the wavelet transform decomposes the signal using a system of so-called wavelets. Compared to the sine waves of the FT that are continuous in time, the different wavelets are localized in both the time and frequency spaces and consist of shifted and scaled copies of the so-called mother wavelet. Even though the short-time Fourier transform is similar to the wavelet transform, in that it is also time and frequency localized, the issues with the former related to the frequency-time resolution trade-off [29] motivate our choice for a wavelet approach. In this work, the continuous wavelet transform (CWT) is applied using the complex Morlet wavelet as mother wavelet, which is characterized by the bandwidth parameter $\gamma_{b}$ and the wavelet center frequency $\gamma_{c}$. For more details about the CWT, the reader is invited to refer to Refs. [24,30]. For the present results, the center frequency is set at $\gamma_{c}=1 \mathrm{~Hz}$, which is in the middle between the two peak frequencies found by the FT $\left(f_{10}\right.$ and $\left.f_{01}\right)$, while $\gamma_{b}=5$ proved to be a reasonable compromise between a sufficient resolution in both the time and frequency domain. In Sec. IV B, the wavelet power spectral density $\left(\operatorname{Psd}_{\mathrm{CWT}}\right)$ will be shown, which is analogous to the $\operatorname{Psd}_{\text {FT }}$ (derived by the FT) but computed as the squared magnitude of the wavelet coefficients $\left|W_{i}\right|^{2}$. 


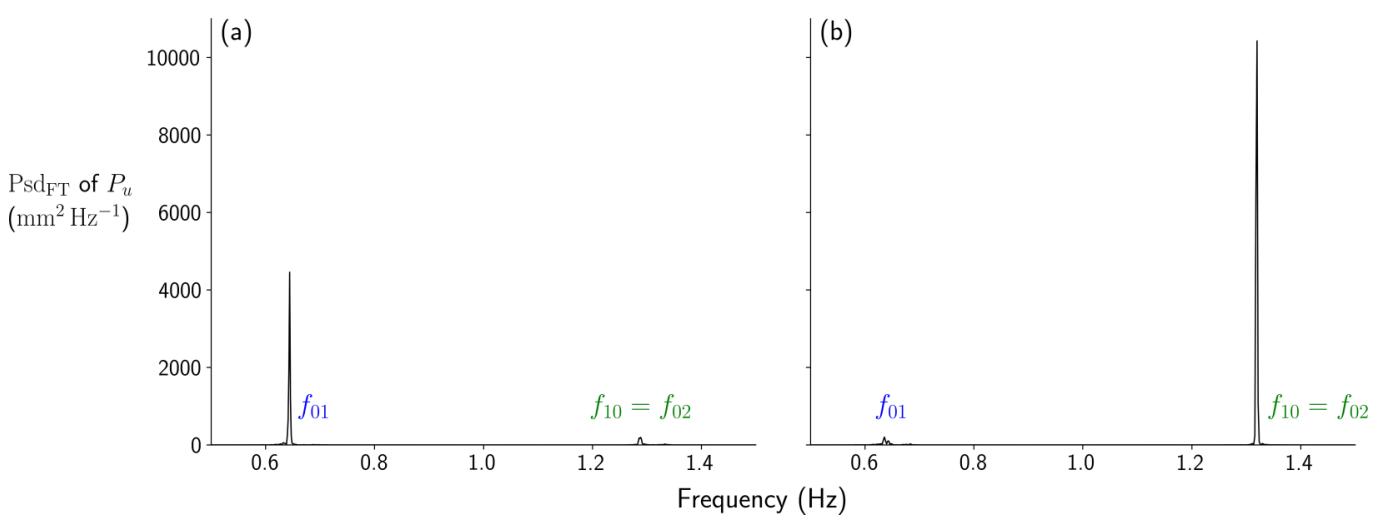

FIG. 2. Psd $\mathrm{FT}_{\mathrm{FT}}$ of the upstream pressure sensor registrations $\left(P_{u}\right)$ for two measurement periods during the test with $\mathrm{Fr}=0.63$, in which the surface predominantly oscillates according to either the transverse $m_{01}$ mode (a) or the longitudinal $m_{10}$ mode (b).

\section{RESULTS AND DISCUSSION}

\section{A. Fourier analysis}

Figure 2 presents the Psd $\mathrm{FT}_{\mathrm{F}}$ of the upstream pressure sensor registrations for two separate time windows during the test with $\mathrm{Fr}=0.63$, in which Fig. 2(a) and Fig. 2(b) correspond to a measurement window with pronounced excitation of the transverse mode $m_{01}$ and the longitudinal mode $m_{10}$, respectively. In both spectra, two peaks can be identified, which approximately match the theoretical frequencies estimated with Eq. (3) for $f_{01}$ and $f_{10}=f_{02}$.

Since the theoretical frequency of $m_{02}$ and $m_{10}$ are equal, the peak at $f_{10}=f_{02} \approx 1.3 \mathrm{~Hz}$ could correspond to either transverse waves (with $n_{t}=2$ nodes over the combined main channel + cavity width $L_{t}=b+W$ ) or to longitudinal waves (with $n_{l}=1$ node over the cavity length $L_{l}=L$ ). Therefore, two additional tests are done at well-chosen Froude numbers (last two rows of Table I), in which the ultrasonic gauge is positioned near the main channel wall opposite to the cavity $\left[U_{2}\right.$ in Fig. 1(b)]. $U_{2}$ corresponds to the location of an antinode of the transverse wave of mode $m_{02}$, while being located outside the area in which longitudinal seiching waves $\left(m_{10}\right)$ are excited (i.e., the cavity). This showed that for a measurement period with a strong peak at $f_{10}=f_{02}$, the surface oscillations at $U_{2}$ are negligible compared to the surface oscillations within the cavity measured by the pressure sensors (not presented in this paper). Therefore, the peak at a frequency equal to $f_{10}=f_{02}$ in Fig. 2(a) and Fig. 2(b) corresponds to a wave that is oscillating longitudinally and which is bounded by the cavity area [confirming the mode $m_{10}$ displayed in Fig. 1(b)]. Moreover, for a frequency of $1.3 \mathrm{~Hz}$, the cross spectrum between the upstream and downstream pressure sensor (not shown in this paper) resulted in a phase difference of $180^{\circ}$, which matches the theoretical shape of the $m_{10}$ mode depicted in Fig. 1(b). This methodology finally permitted to conclude that mode $m_{01}$ is dominant for the time period considered in Fig. 2(a), while $m_{10}$ is dominant for the time period of Fig. 2(b) and that mode $m_{02}$ does not take place.

Figure 3 shows the amplitude $\Delta \eta_{\mathrm{FT}}$ for the two most energetic modes of oscillation $\left(m_{10}\right.$ and $m_{01}$ ) in function of the Froude number of the main stream, where $\Delta \eta_{\mathrm{FT}}$ is made dimensionless by the average head in the main stream (i.e., $h_{m}+U_{b}^{2} / 2 g$ ).

The results in Fig. 3 confirm the general trend reported in Refs. [12,20,22], i.e., pronounced seiching is only excited for sufficiently high Froude numbers $(\mathrm{Fr}>0.5-0.55)$ and the dominant mode of seiching changes from transverse $\left(m_{01}\right)$ to longitudinal $\left(m_{10}\right)$ seiching with increasing Froude number. For the present flow configurations $\left(h_{m} / L \simeq 0.36\right)$, the dominant mode of seiching changes around $\mathrm{Fr}=0.6$, after which for increasing Froude numbers $(\mathrm{Fr}>0.6)$ the amplitude of $m_{10}$ increases and the amplitude of $m_{01}$ decreases. However, in contrast with previous studies where 


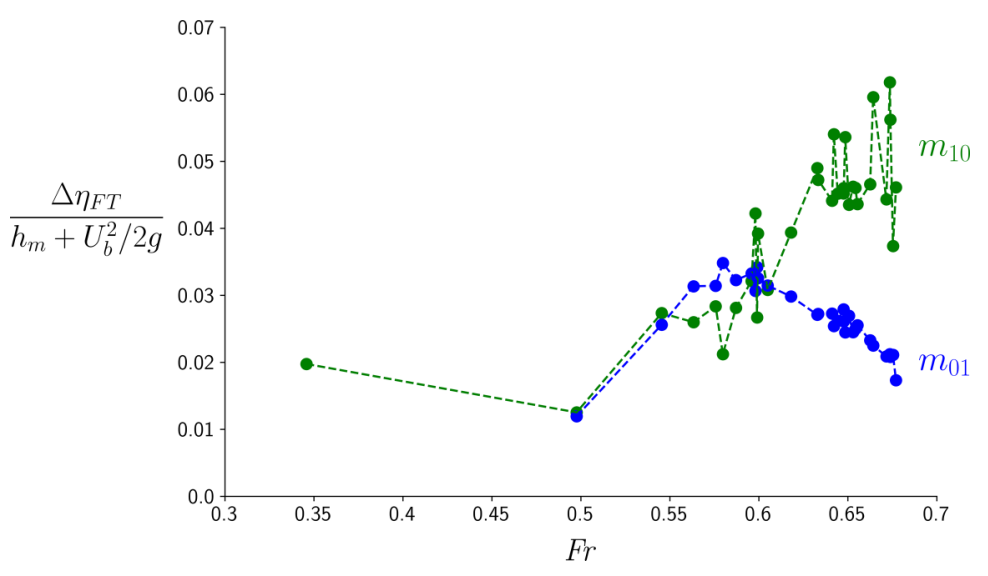

FIG. 3. Nondimensional amplitude of the seiching modes $m_{01}$ and $m_{10}$ in function of the Froude number of the main stream, in which the seiching amplitudes are estimated by integration of the $\operatorname{Psd}_{\mathrm{FT}}$.

the amplitude of seiching was also quantified in function of Fr [12,15,18,20,22], the amplitudes of the seiching modes depicted in Fig. 3 do not follow a smooth trend. Herewith, especially $\Delta \eta_{\mathrm{FT}, m_{10}}$ (green symbols) fluctuates significantly for varying Froude number. It is still unclear what causes the amplitudes of a single mode to vary significantly for a negligible change in Froude number (compared to the literature). However, Fig. 2 showed that the total measurement time $(90 \mathrm{~min})$ is actually composed of consecutive time windows in which either transverse $\left(m_{01}\right)$ or longitudinal $\left(m_{10}\right)$ seiching is dominant. This intermittent behavior of seiching was confirmed by visual analysis of the time series of the different tests. Therefore, a wavelet transform is in this work adopted as an alternative strategy to analyze the signal in both the time and frequency domains (Sec. IV B).

\section{B. Wavelet analysis}

Figure 4 shows the Psd ${ }_{\text {CWT }}$ in function of time $t$ and frequency $f$ (both made nondimensional by $f_{10}$ ) for the test with $\mathrm{Fr}=0.64$ (similar Froude number as $\mathrm{Fr}=0.63$ in Fig. 2). The two main characteristic frequencies of the cavity $\left(f_{01}\right.$ and $\left.f_{10}\right)$ can be distinguished in the wavelet spectrum as horizontal regions with a high wavelet power. Similarly to Fourier spectra (Fig. 2), a high wavelet power indicates that the corresponding oscillation mode (standing waves at a particular frequency) has a large, instantaneous energy and thus corresponds to standing waves with a large amplitude for that specific time instance. The zoomed-in view of the Psd $\mathrm{CWT}_{\mathrm{CW}}$ during the dimensionless time window $t f_{10}=$ [3000-3250], indicated by a red dashed line in Fig. 4, suggests that the energy corresponding to each mode can vary rapidly in a short time period. In the Supplemental Material [31], a video of the surface oscillations inside the cavity is given for a time window in which the dominant seiching mode switches from transverse to longitudinal seiching. Moreover, it indicates that the magnitude of $m_{10}$ and $m_{01}$ are inversely related, even though both modes can (partly) exist simultaneously (nonzero wavelet coefficients for both frequencies at the same time).

Based on the Psd ${ }_{\mathrm{CWT}}$ in Fig. 4, it is possible to perform a frequency scale decomposition of the time series. For this purpose, the inverse wavelet transform is applied to convert Fig. 4 back to the time domain in which the longitudinal $m_{10}$ and transverse $m_{01}$ mode are reconstructed separately. Herewith, the wavelet power Psd ${ }_{C W T}$ is evaluated in a frequency band with a width of $0.6 \mathrm{~Hz}$ centered around either the peak frequencies $f_{01}$ or $f_{10}$ for the modes $m_{01}$ or $m_{10}$, respectively. Figure 5 presents the reconstructed and decomposed time series of the surface registrations of $P_{u}$. These results confirm the intermittent character of the wavelet power in Fig. 4, in which time windows with a large $\operatorname{Psd}_{\mathrm{CWT}}$ at frequencies $f_{01}$ and $f_{10}$ correspond to time windows with a large amplitude of modes $m_{01}$ (blue in Fig. 5) and $m_{10}$ (green in Fig. 5), respectively. Additionally, the 


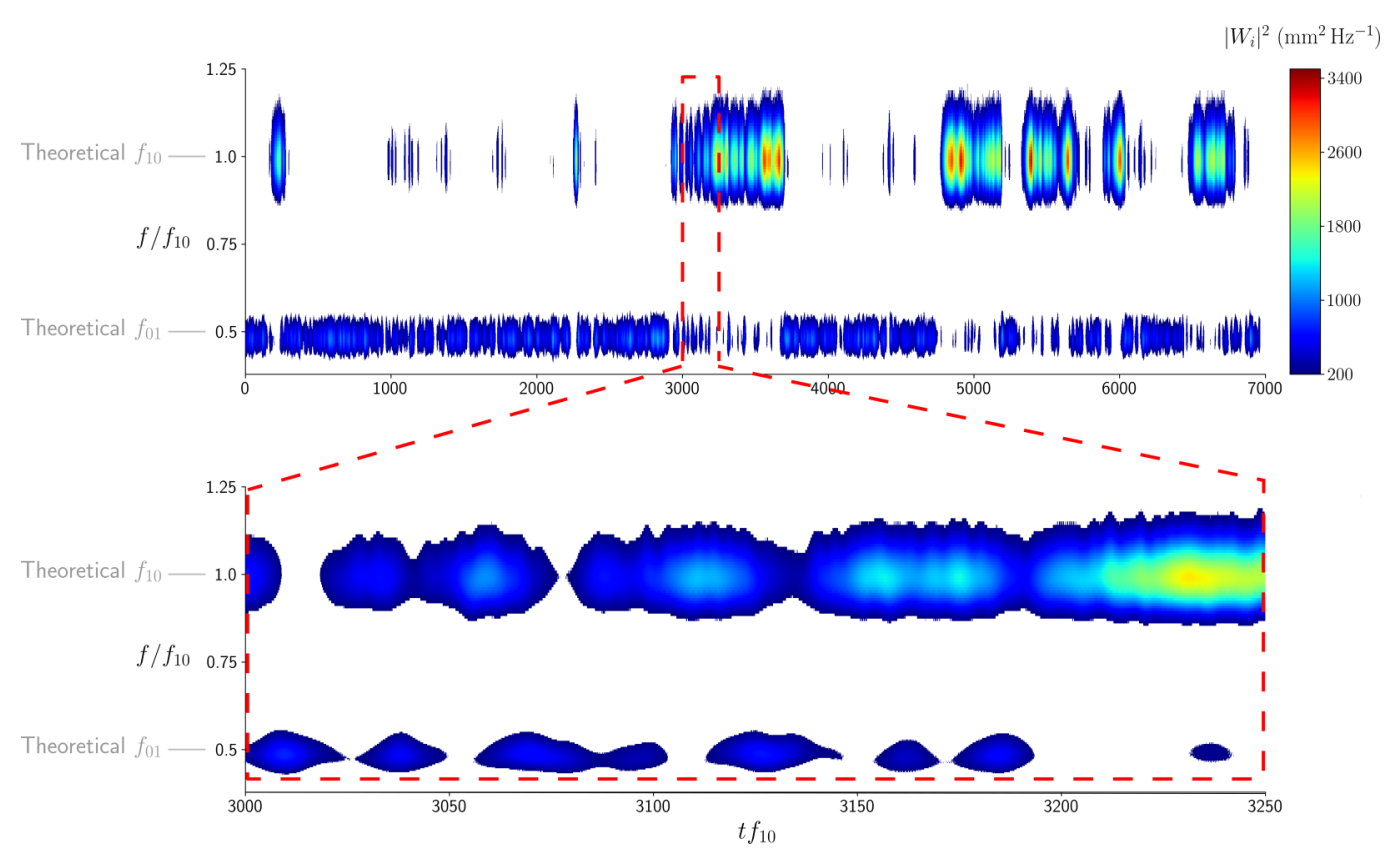

FIG. 4. Psd $\mathrm{CWT}_{\mathrm{T}}$ in function of time and frequency for the test with $\mathrm{Fr}=0.64$, with a zoomed-in view of the $\operatorname{Psd}_{\mathrm{CWT}}$ during the dimensionless time window $t f_{10}=[3000-3250]$.

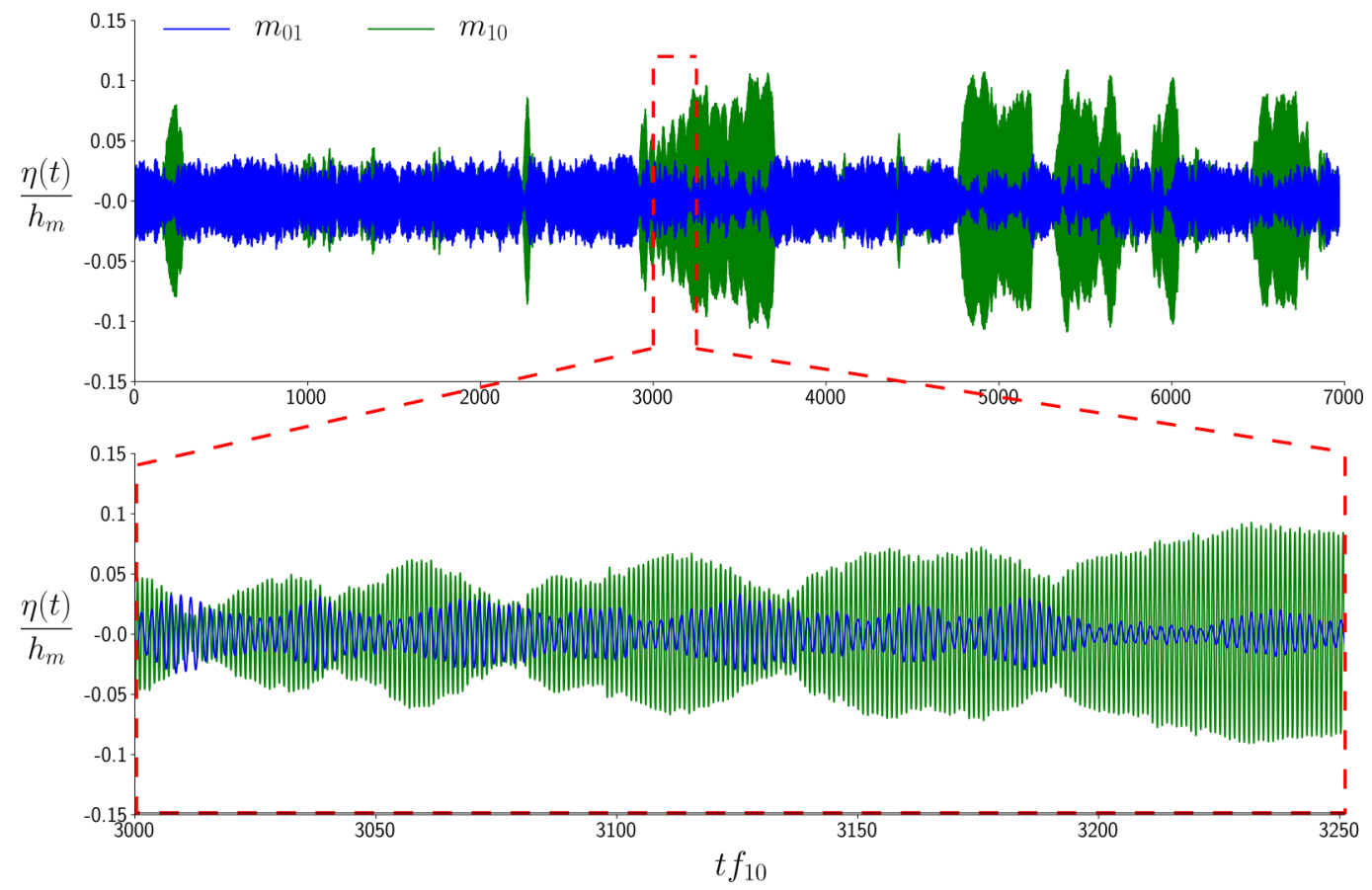

FIG. 5. Reconstructed and decomposed time series $\left[\eta(t) / h_{m}\right]$ of the test with $\mathrm{Fr}=0.64$ by the upstream pressure sensor $P_{u}$, in which the surface is reconstructed by the inverse wavelet transform for each seiching mode separately ( $m_{01}$ in blue and $m_{10}$ in green). In agreement with Fig. 4, a zoomed-in view of the reconstructed and decomposed time series is presented during the dimensionless time window $t f_{10}=[3000-3250]$. 


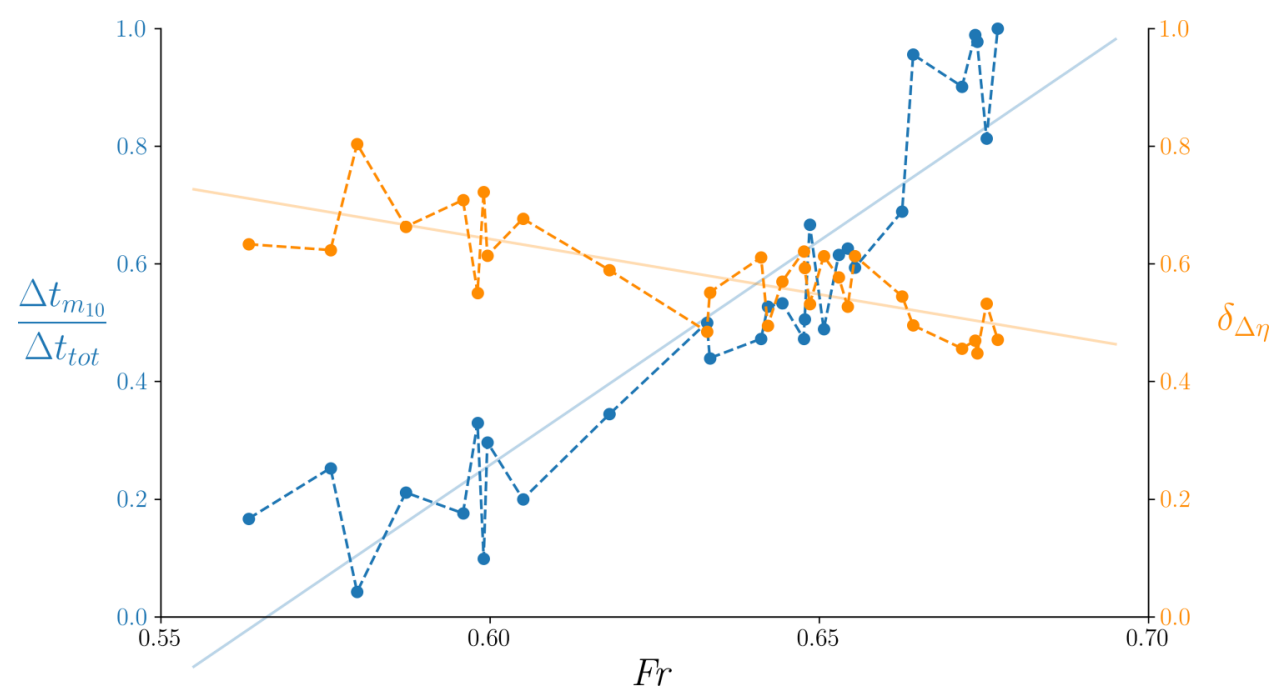

FIG. 6. Relative duration of dominance of mode $m_{10}$ (left axis in blue: $\Delta t_{m_{10}} / \Delta t_{\text {tot }}$ ) and relative difference between FT and CWT amplitudes (right axis in orange: $\delta_{\Delta \eta}$ ) in function of the Froude number of the main stream. For both graphs, the best-fit straight line through the data between $\mathrm{Fr}=0.56-0.68$ (used for the results in Fig. 8) is depicted semitransparent.

zoomed-in view in Fig. 5 for the time window $t f_{10}=$ [3000-3250] (indicated by a red dashed line) further substantiates that a decrease in the amplitude of standing waves of mode $m_{01}$ result in an increase of the amplitude of $m_{10}$ waves and vice versa.

In contrast to Figs. 4 and 5 , the amplitudes $\Delta \eta_{\mathrm{FT}}$ (Fig. 3) are determined by integration of the Psd ${ }_{\mathrm{FT}}$ that is computed from the entire (unsteady) time series. The energy or power $\mathrm{P}_{\mathrm{FT}}$ thus corresponds to the average power of $m_{10}$ or $m_{01}$ over the entire measurement period (90 min), in which the magnitude of each $\operatorname{Psd}_{\mathrm{FT}}$ (and thus of $\mathrm{P}_{\mathrm{FT}}$ ) is linearly scaled by the relative duration in which the corresponding individual mode is excited. For example, if standing waves of a single mode are only triggered during $60 \%$ of the measurement time, then $\Delta \eta_{\mathrm{FT}}$ will underestimate the physical amplitude of the waves by a factor $\sqrt{0.6}$. Therefore, an alternative strategy seems more appropriate to derive an estimate that is representative for the physical amplitude of the seiching waves.

For this purpose, we compute the amplitude corresponding to $m_{01}$ and $m_{10}$ in consecutive time windows of $60 \mathrm{~s}$ (vertical bands in Figs. 4 and 5), such that for every test lasting $90 \mathrm{~min}, 90$ consecutive so-called $\Delta \eta_{i, m}$ are derived ( $i=1$ to $90, m=m_{01}$ or $m_{10}$ ). Denoting $t_{m_{10}}$ as the time windows for which longitudinal seiching is dominant $\left(\Delta \eta_{i, m_{10}}>\Delta \eta_{i, m_{01}}\right)$, the dominance of mode $m_{10}$ is quantified by the ratio $\Delta t_{m_{10}} / \Delta t_{\text {tot }}$ in which $\Delta t_{m_{10}}$ equals the combined duration of $t_{m_{10}}$ and $\Delta t_{\text {tot }}$ equals the total measurement time $(90 \mathrm{~min})$. As could be expected from Fig. 3 , the increasing trend of $\Delta t_{m_{10}} / \Delta t_{\text {tot }}$ in Fig 6 (left axis in blue) shows that the longitudinal seiching mode $m_{10}$ becomes more frequently excited with increasing Froude number. Note that the right axis of Fig. 6 (orange) will be introduced later in this paper.

Figure 6 indicates a range of Fr where $\Delta t_{m_{10}}$ and $\Delta t_{m_{01}}$ are of the same order of magnitude, i.e., where $\Delta t_{m_{10}} / \Delta t_{\text {tot }}$ is significantly larger than 0 but also significantly lower than 1 . Therefore, the measurements seem to be composed of alternating time windows with either $m_{10}\left(t_{m_{10}}\right)$ or $m_{01}$ $\left(t_{m_{01}}\right)$ being dominant. For this reason, instead of quantifying $\Delta \eta_{\mathrm{FT}}$ based on the entire time series (as in Fig. 3), this work employs two alternative and more time-localized strategies to quantify the magnitude of each seiching mode using the amplitudes $\Delta \eta_{i, m}$ determined in every interval:

(i) $\Delta \eta_{\max }$ : the maximum value of $\Delta \eta_{i, m_{01}}$ and $\Delta \eta_{i, m_{10}}$ in a single test; 


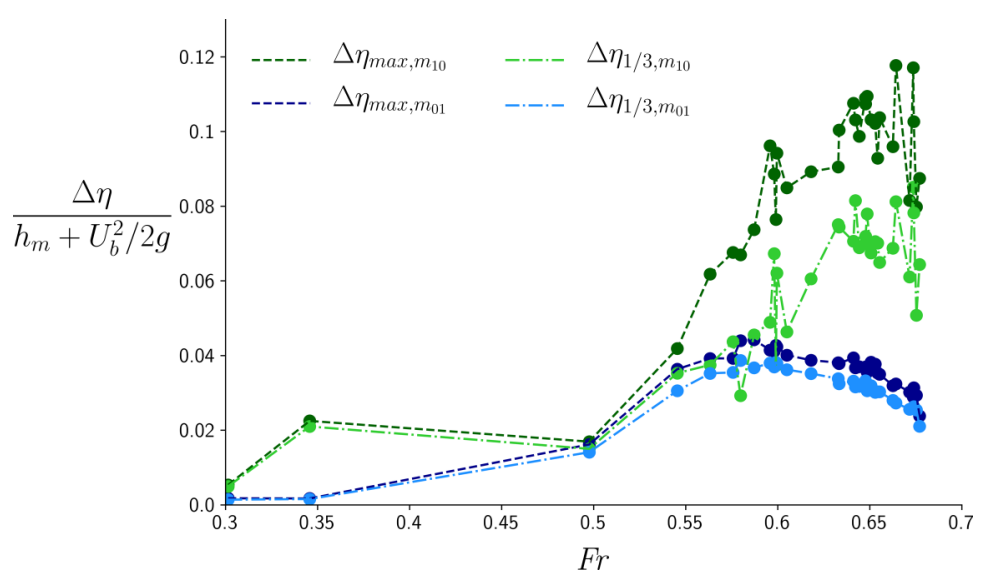

FIG. 7. Nondimensional amplitude of the seiching modes in function of the Froude number of the main stream in which both $\Delta \eta_{\max }$ and $\Delta \eta_{1 / 3}$ are displayed.

(ii) $\Delta \eta_{1 / 3}$ : the mean value of the highest one-third (33\%) of $\Delta \eta_{i, m_{01}}$ and $\Delta \eta_{i, m_{10}}$ (separately) in a single test.

Figure 7 shows $\Delta \eta_{\max }$ and $\Delta \eta_{1 / 3}$ for mode $m_{10}$ and $m_{01}$ in function of the Froude number of the main stream. In general, the wavelet results confirm that the magnitude of the longitudinal seiching waves ( $m_{10}$, green symbols) increases with increasing Froude numbers, while the magnitude of transverse seiching waves ( $m_{01}$, blue symbols) decreases for $\mathrm{Fr}>0.6$ with increasing Froude number. Moreover, the fluctuating trend of $\Delta \eta_{\mathrm{FT}, m_{10}}=f(\mathrm{Fr})$ in Fig. 3 seems less pronounced in Fig. 7 , in which especially for $\Delta \eta_{\max , m_{10}}$ a more gradual increase with increasing Froude number can be observed.

To confirm our hypothesis that the difference between the FT and CWT estimates is caused by the limited time in which each mode is excited, Figure 6 also shows the relative difference between the two estimated amplitudes $\delta_{\Delta \eta}=\left(\Delta \eta_{\max , m_{10}}-\Delta \eta_{\mathrm{FT}, m_{10}}\right) / \Delta \eta_{\max , m_{10}}$ in function of the Froude number of the main stream (orange results on the right axis). Comparing both graphs in Fig. 6, a local minimum of the relative duration $\Delta t_{m_{10}} / \Delta t_{\text {tot }}$ corresponds with a local maximum of $\delta_{\Delta \eta}$. To better illustrate this inverse relationship, we determine for both curves in Fig. 6 the deviation with their best-fit straight line through the data between $\mathrm{Fr}=0.56-0.68$ (light colored lines in Fig. 6). Figure 8 shows these deviations with respect to the linear trend for $\Delta t_{m_{10}} / \Delta t_{\text {tot }}$ and the negative of the deviations for $\delta_{\Delta \eta}$. The close resemblance between the irregularities of the two curves in Fig. 8 confirms our hypothesis that $\Delta \eta_{\mathrm{FT}}$ is biased by the intermittent character of the selected dominant seiching mode.

Although not presented in this paper, additional analysis revealed that the duration of dominance by either $m_{10}$ or $m_{01}$ varies significantly, ranging from less than $1 \mathrm{~min}$ to the entire measurement period $\left(90 \mathrm{~min}\right.$ ) depending on the flow case. For example, in Fig. 4, periods of dominance of $m_{01}$ (average duration $126 \mathrm{~s}$ ) typically last longer than those of $m_{10}$ (average duration $77 \mathrm{~s}$ ), while both are still significantly larger than the natural periods of seiching $\left(1 / f_{10}\right.$ and $1 / f_{01}: 0.75$ and $1.5 \mathrm{~s}$ ). Moreover, no clear periodicity in the alternating periods of dominant $m_{10}$ or $m_{01}$ could be distinguished, which should therefore further be investigated in future research. For this purpose, surface registrations could be combined with simultaneous velocity measurements.

\section{Time-series analysis}

In this last section, the surface oscillations are investigated in the time domain to deepen our understanding on the mode switching behavior from $m_{01}$ to $m_{10}$ or vice versa. As explained in Sec. II, the current experimental geometry $(b=W=L)$ causes $f_{01}$ to be (approximately) half of $f_{10}$, 


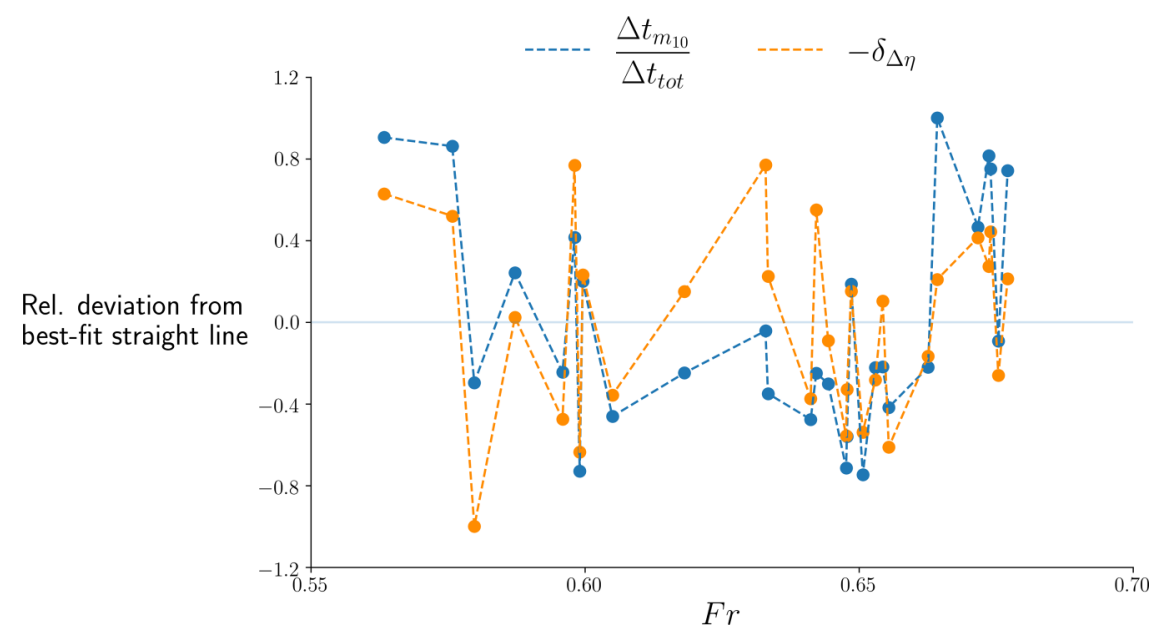

FIG. 8. Relative deviation of $\Delta t_{m_{10}} / \Delta t_{\text {tot }}$ and the negative of the relative deviation of $\delta_{\Delta \eta}$ in Fig. 6 from the corresponding best-fit straight line (Fig. 6). The resembling shape of both curves confirms that $\Delta t_{m_{10}} / \Delta t_{\text {tot }}$ and $\delta_{\Delta \eta}$ are inversely related.

which enables both types of standing waves to coexist at a single forcing frequency. For seiching to be excited, Refs. $[3,12,23]$ showed that the energy is transferred from the forcing mechanism (periodic vortex shedding) to the surface oscillations by impingement of the vortices onto the downstream corner near $U_{1}$. The vortex impingement and corresponding rise of the local water depth at the downstream cavity wall should therefore be in a constant phase with the seiching waves to systematically feed energy to the seiching waves. As such, it is reasonable to assume that the phase difference between the transverse and longitudinal waves at the inner corners of the cavity will not be arbitrary in case both receive energy from the periodic forcing.

This section describes the relative phase of the transverse waves (with frequency $f_{01}$ ) and the longitudinal waves (with frequency $f_{10} \approx 2 f_{01}$ ) and compares the resulting theoretical surface variations with the experimental time recordings of the ultrasonic gauge $\left(U_{2}\right)$ and the pressure sensors $\left(P_{u}\right.$ and $\left.P_{d}\right)$ for the flow case with $\mathrm{Fr}=0.64$.

Figure 4 showed that most of the time, one mode dominates the other. Moreover, if one only considers situations where the waves according to modes $m_{10}$ and $m_{01}$ reach their maximum amplitude simultaneously, then two possible states of superposition at that specific location can arise:

(i) State CC (crest-crest)

In state $\mathrm{CC}$, the crests of the longitudinal wave (with frequency $f_{10}=2 f_{01}$ ) coincide (in time, i.e occurs at the same time as) with the crests and troughs of the transverse wave (with frequency $f_{01}$ ), see Fig. 9(a).

(ii) State TT (trough-trough)

State TT corresponds to the situation in which the troughs of the longitudinal wave (with frequency $f_{10}=2 f_{01}$ ) coincide with the crests and troughs of the transverse wave (with frequency $f_{01}$ ), see Fig. 9(b).

For two artificial sine waves of frequencies $f_{01}$ and $f_{10}=2 f_{01}$, Fig. 9 depicts the two aforementioned superposition states CC (a) and TT (b). To enable better comparison with the experimental data (see further), the mean water depth for the artificial time series is chosen equal to $h_{m}=14.5 \mathrm{~cm}$ and the amplitude of the two artificial sine waves (green and blue) is based on the experimentally determined amplitudes $\Delta \eta_{\mathrm{FT}}$ of the measured seiching modes $\left(m_{01}\right.$ and $\left.m_{10}\right)$ during a time window where $m_{01}$ (blue dash curve) is dominant, i.e., $t_{m_{01}}$ where $\Delta \eta_{i, m_{01}}>\Delta \eta_{i, m_{10}}$. 

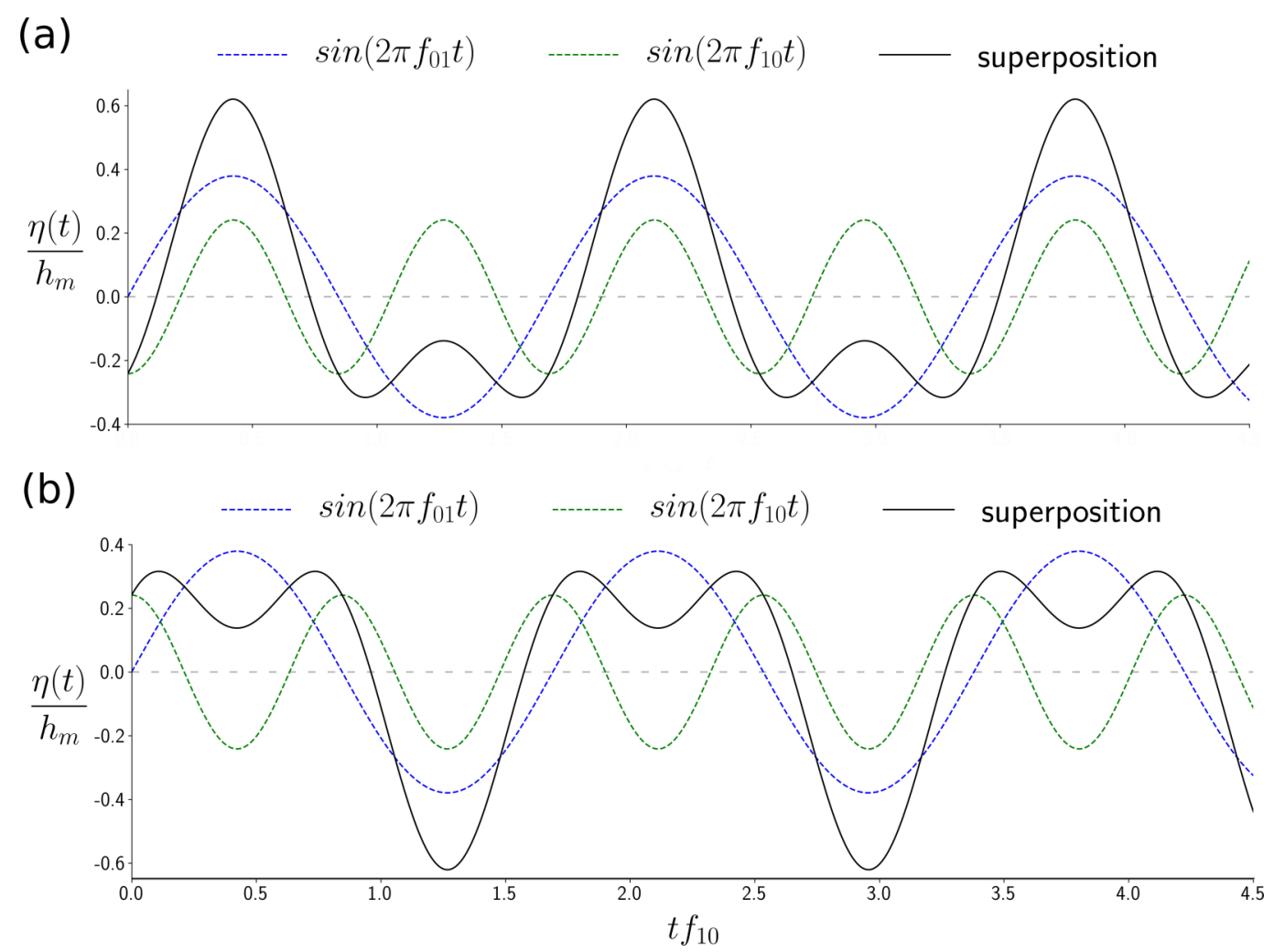

FIG. 9. Depending on the relative phase between the artificial sine waves with frequency $f_{01}$ and frequency $f_{10}=2 f_{01}$, the superimposed surface variation during a time period with dominant mode $m_{01}\left(t_{m_{01}}\right)$ is characterized by a reciprocal succession of small and large crests or small and large troughs for state CC (a) and state TT (b), respectively.

In theory, a similar approach could be used for the time windows where $m_{10}$ dominates, i.e., $t_{m_{10}}$ where $\Delta \eta_{i, m_{10}}>\Delta \eta_{i, m_{01}}$. In practice, however, the influence of $m_{01}$ during $t_{m_{10}}$ remains too small to reliably distinguish higher order influences in the sine waves (with frequency $f_{10}$ ) of the recorded time series. Therefore, this work will only further discuss the superposition $m_{01}$ and $m_{10}$ during $t_{m_{01}}$ periods.

The superposition of the two artificial sines in Fig. 9(a) (black) shows that in state CC, the global surface variation is characterized by periodically alternating small and large crests, while all troughs reach the same value of $\eta$ [i.e., $\eta(t) / h_{m} \simeq-0.3$ ]. The opposite takes place for state TT: The superposition depicted in Fig. 9(b) (black) is characterized by crests of equal magnitude [i.e., $\eta(t) / h_{m} \simeq 0.3$ ] and troughs of periodically alternating large and small magnitude. The next two subsections will analyze two possible scenarios for the actual free-surface deformation in the cavity in case the $m_{01}$ and $m_{10}$ waves reach their maximum amplitude simultaneously in the inner corners of the cavity (close to $P_{u}$ and $P_{d}$ ), i.e., either state TT at the inner upstream corner and state CC at the inner downstream corner (denoted scenario A) or vice versa (denoted scenario B). Figure 10 gives a graphical representation of these two possible scenarios A and B, in which instantaneous surface deformations according to a single mode but with a phase difference of $180^{\circ}$ are distinguished from each other by their line style (full or dashed line).

\section{Scenario A}

In scenario A (orange color in Fig. 10), the surface evolution in time at the downstream inner corner of the cavity is characterized by state $\mathrm{CC}$, while the surface evolution at the upstream inner 


\section{plan view}

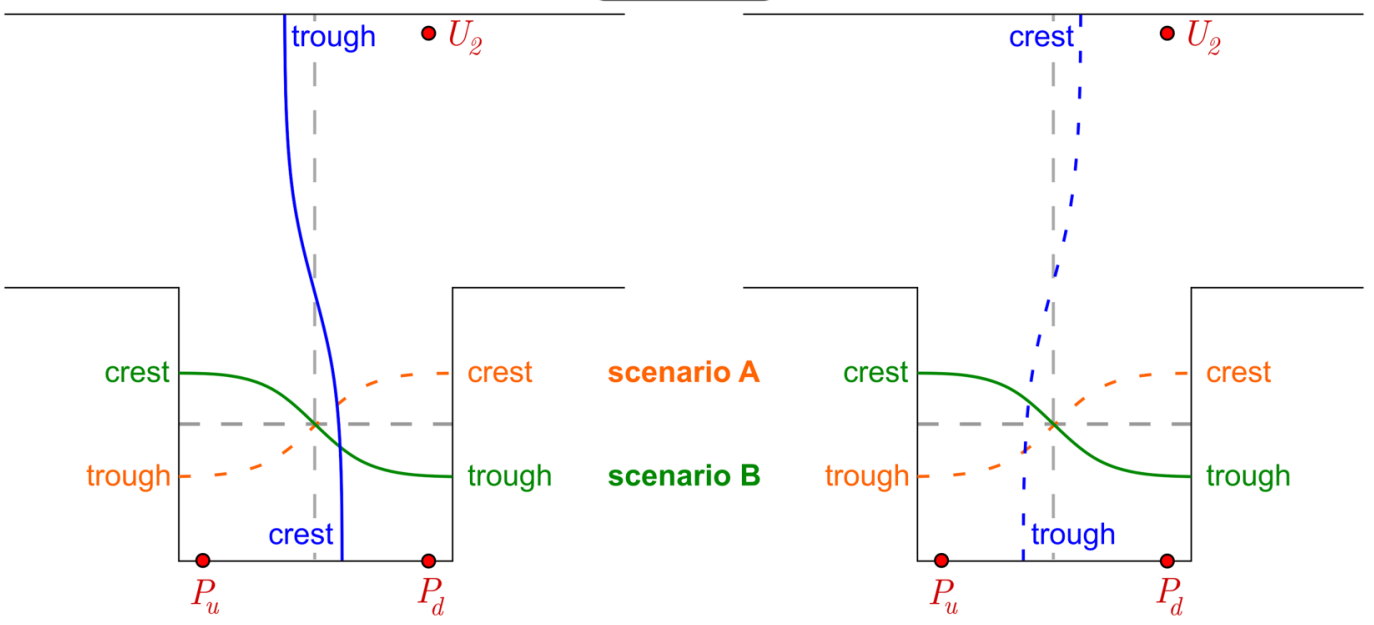

FIG. 10. Sketch of the two possible scenarios (scenarios A and B) in case the waves corresponding to $m_{10}$ and $m_{01}$ reach their maximum amplitude simultaneously in the inner corners of the cavity (near $P_{u}$ and $\left.P_{d}\right)$. Instantaneous surface deformations according to a single mode but with a phase difference of $180^{\circ}$ are distinguished from each other by their line style (full or dashed line).

corner of the cavity corresponds to state TT. Because $P_{u}$ and $P_{d}$ are located close to the inner corners of the cavity, the (experimental) pressure recordings during scenario A should match Fig. 9(a) and Fig. 9(b) for the downstream $\left(P_{d}\right)$ and upstream $\left(P_{u}\right)$ pressure sensor, respectively. Therefore, the typical theoretical alternation of large and small crests (or troughs) in the experimental recordings of the pressure sensors for the flow case with $\mathrm{Fr}=0.64$ (ultrasonic gauge positioned at $U_{2}$ ) is used to determine time periods corresponding to scenario A or scenario B. Figure 11 gives a typical example of the measured time series $\left[\eta(t) / h_{m}\right]$ during a time period that is identified as scenario $\mathrm{A}$, in which the orange and brown vertical lines indicate the time instances with crests of the longitudinal waves of mode $m_{10}$. Given that $U_{2}$ is located at the opposite side of the cavity (along the main stream side wall), a high (orange line) and low (brown line) water level at $U_{2}$ correspond to, respectively, a trough (orange line) and crest (brown line) of the transverse wave of mode $m_{01}$ at the inner cavity wall.

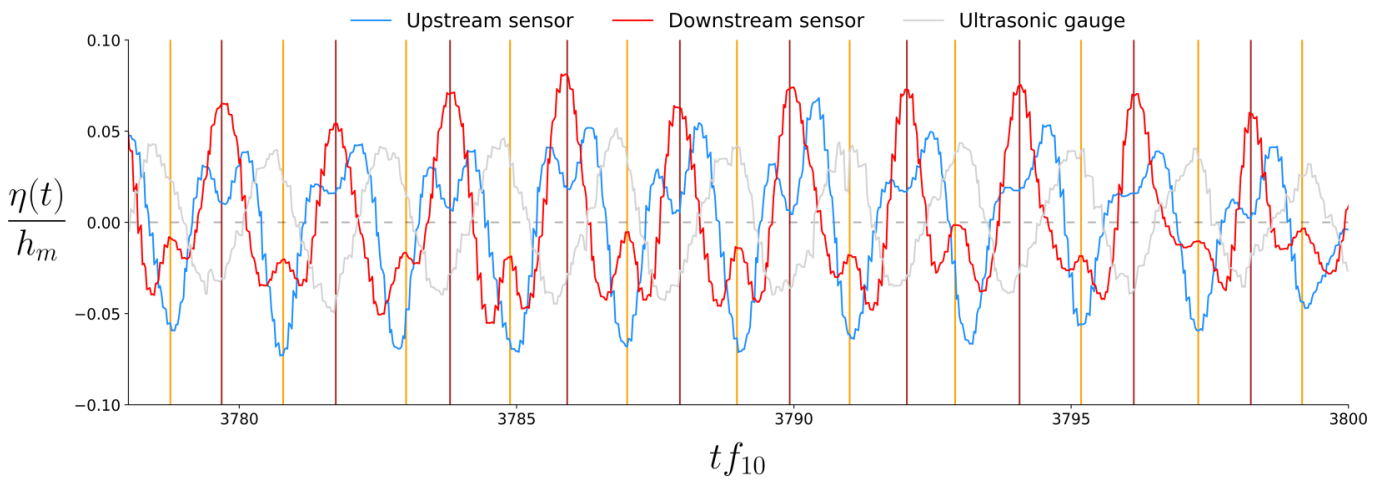

FIG. 11. Recorded time series $\left[\eta(t) / h_{m}\right]$ of the test with Fr $=0.64$ during a time window in which $m_{01}$ dominates $\left(t_{m_{01}}\right)$ and the seiching modes have a phase difference determined by state CC in the downstream inner corner of the cavity and by state TT in the upstream inner corner of the cavity, i.e., corresponding to scenario A. 


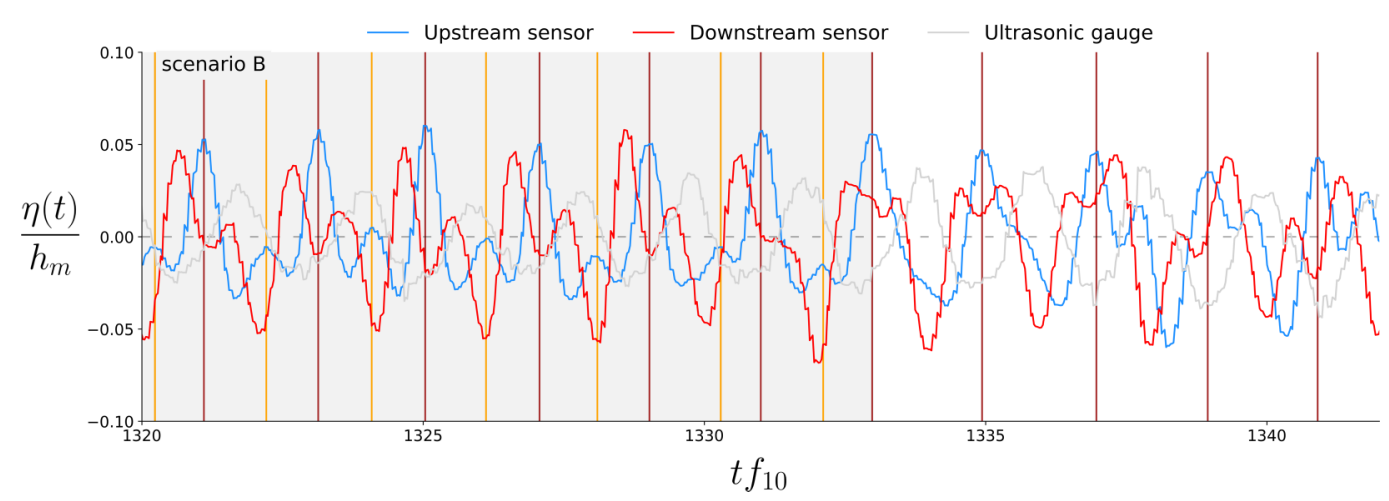

FIG. 12. Recorded time series $\left[\eta(t) / h_{m}\right]$ of the test with $\mathrm{Fr}=0.64$ during a time window in which $m_{01}$ dominates $\left(t_{m_{01}}\right)$ and where, for the time window indicated in light gray, the seiching modes have a phase difference determined by state $\mathrm{CC}$ in the upstream inner corner of the cavity and by state TT in the downstream inner corner of the cavity, i.e., corresponding to scenario B.

Since $U_{2}$ is located outside the area in which longitudinal seiching is excited (i.e., the cavity), the absence of the higher frequency oscillations (with frequency $f_{10}$ ) in the recording of the ultrasonic gauge (gray curve) seems not surprising. Moreover, the recordings of the two pressure sensors match quite well with the theoretical expectations: state CC [as in Fig. 9(a)] and state TT [as in Fig. 9(b)] fairly agree with the surface variation at $P_{d}$ and $P_{u}$, respectively. Applying the template matching technique to the entire time series of the test with $\mathrm{Fr}=0.64$, scenario A could be distinguished during $63 \%$ of the total measurement time in which mode $m_{01}$ dominated the surface shape.

\section{Scenario B}

Opposite to scenario A, scenario B (green color in Fig. 10) corresponds to the situation where state CC can be observed at the upstream inner corner of the cavity (close to $P_{u}$ ) and state TT at the downstream inner corner of the cavity (close to $P_{d}$ ). Using the same methodology as explained before, Figure 12 plots the experimental recordings $\left[\eta(t) / h_{m}\right]$ during a time period identified as scenario B (indicated in light gray). Similarly as in Fig. 11, a high and low water level measured at $U_{2}$ correspond to, respectively, a trough (orange line) and crest (brown line) of the transverse wave of mode $m_{01}$ at the inner cavity wall. In general, the experimental recordings show a reasonable correspondence with the theoretical surface variations depicted in Figure 9, with state CC [Fig. 9(a)] and state TT [Fig. 9(b)] matching the surface variations at $P_{u}$ (blue curve) and $P_{d}$ (red curve), respectively. Note that after the period of scenario B in Fig. 12 (indicated in light gray), an intermediate scenario can be observed in which a gradual shift to scenario A takes place.

For the entire measurement duration of the flow case with $\mathrm{Fr}=0.64$, scenario $\mathrm{B}$ could be distinguished during $18 \%$ of the total measurement time in which mode $m_{01}$ dominated the surface shape. Combining these $18 \%$ of $\Delta t_{m_{01}}$ of scenario B with the $63 \%$ of $\Delta t_{m_{01}}$ of scenario A results in $81 \%$. Therefore, only for $19 \%$ of $\Delta t_{m_{01}}$ neither the crests or troughs of the two different seiching waves are in phase. For those time periods, neither scenario A or scenario B could be identified but rather an intermediate scenario takes place (similarly to the period with $t f_{10}>1332$ in Fig. 12).

While it can be concluded that scenario A and B correspond to two possible yet unstable flow states that alternate throughout time, it is still unclear what determines the selected flow state at one particular time instance. Nonetheless, Ref. [18] also studied the bidirectional seiching phenomenon in a square cavity with $L=W=b$, in which they combined surface measurements with velocity measurements at the main stream-cavity interface. This showed that even if multiple modes with different frequencies characterize the surface shape, a single dominant vortex frequency in the mixing layer is observed. Combined with the results presented in this paper, a single vortex shedding 


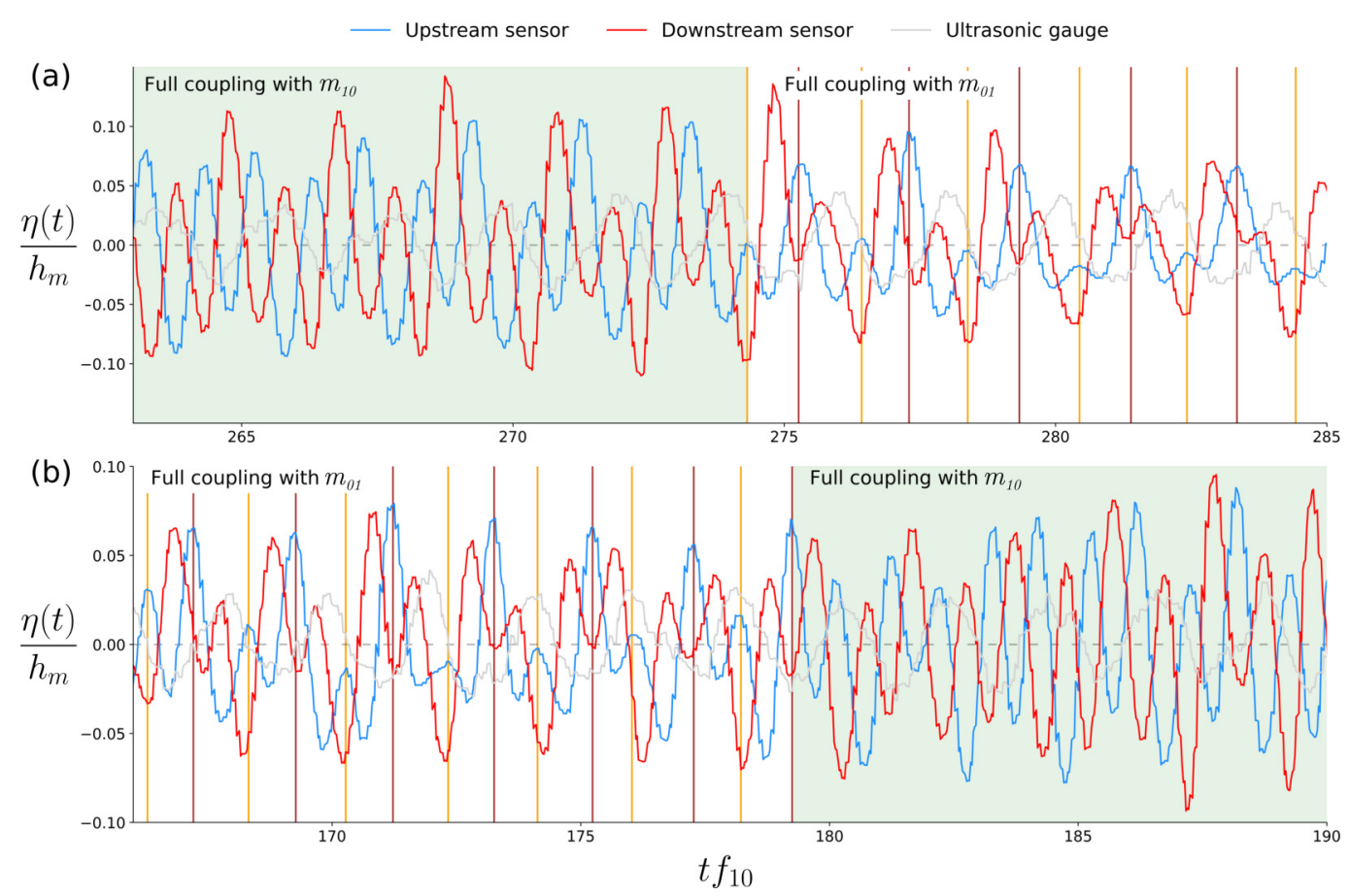

FIG. 13. Recorded time series $\left[\eta(t) / h_{m}\right]$ of the test with $\mathrm{Fr}=0.64$ corresponding to a time window at the start (a) or at the end (b) of a period with the transverse seiching mode $m_{01}$ being dominant $\left(t_{m_{01}}\right)$, in which scenario B can be distinguished just after the end (a) or prior to the start (b) of a period where mode $m_{10}$ is dominant $\left(t_{m_{10}}\right.$, indicated in green).

behavior at a fixed frequency can thus excite different seiching modes and different superposition states (scenario A or B). Herewith, the results of Ref. [18] showed that the vortex shedding frequency is fully in phase with the lowest seiching frequency $\left(f_{01}\right)$, while the higher frequencies of other seiching modes $\left(f_{10}=f_{02}, \ldots,\right)$ are a multiple of this fundamental frequency. The impingement on the downstream corner and following entrainment into the cavity of the mixing layer vortices thus provides energy to two seiching modes, in which the relative energy distribution between the transverse and longitudinal modes can vary during the same experiment. In case of mode $m_{10}$ and $m_{02}$ at $f_{10}=f_{02}$, energy input from the mixing layer vortices to the seiching free-surface oscillations only occurs every two periods of the standing waves.

Additionally, detailed analysis showed that scenario B can always be observed at the start and at the end of a period with $m_{01}$ being dominant $\left(t_{m_{01}}\right)$, for which an example is shown in Fig. 13(a) and Fig. 13(b), respectively. This behavior was confirmed by other transitions from $t_{m_{10}}$ to $t_{m_{01}}$ (or vice versa) for the same test (with Fr $=0.64$ ) or for tests at different Froude numbers (not shown), in which the same typical transition between scenario B and a period $t_{m_{01}}$ could almost always be distinguished. Nonetheless, scenario B appeared to be only a necessary but not a sufficient condition for a transition of the dominant seiching mode to occur. This is illustrated by Fig. 12, in which, after a period where scenario B is distinguished, the surface remains dominated by the $m_{01}$ mode and the free-surface oscillations gradually evolve back to scenario A.

\section{CONCLUSIONS}

Present experimental laboratory study was devoted to the so-called bidirectional seiching phenomenon, i.e., sustained oscillations of the free surface in a lateral, open-channel cavity, for 
the specific case where the length of the cavity equals half of the combined width of the cavity and the main channel. This characteristic geometry caused that standing gravity waves in both the longitudinal $\left(m_{10}\right)$ and transverse $\left(m_{01}\right)$ direction were excited simultaneously. The bidirectional aspect of seiching was studied in function of the Froude number of the main stream for a fixed geometry and water depth. To that end, the frequency and (relative) amplitude of the seiching waves were determined based on the power spectral density of the experimentally recorded surface oscillations, which showed that the dominance of longitudinal seiching (mode $m_{10}$ ) grows with increasing Froude number.

Second, a wavelet analysis was applied to investigate the wave energy in both the time and frequency domains. This indicated that the amplitude of both modes may fluctuate significantly during the course of a single experiment. As such, the total measurement time could be subdivided in periods in which one of the two modes dominated the surface shape. The wavelet results confirmed that the dominance of the $m_{10}$ mode grows with increasing Froude number (i.e., a larger part of the total measurement time dominated by $m_{10}$ ). Moreover, alternative and more time-localized strategies were presented to quantify the time-varying magnitude of the seiching modes and to derive an indicator that is less affected by the intermittent character of seiching compared to the traditional Fourier approach.

Finally, a more in-depth analysis of the raw time series for the one selected flow case with $\mathrm{Fr}=0.64$ showed that the bidirectional seiching waves should fulfill a certain phase criterion to be excited simultaneously. Even though only the time periods where the $m_{01}$ mode dominates could be discussed, the present results showed that the two types of standing waves $\left(m_{01}\right.$ and $\left.m_{10}\right)$ reach either their crests or troughs at the same time in the inner corners of the cavity during more than $80 \%$ of the measurement time. Moreover, for a transition from dominant $m_{01}$ to dominant $m_{10}$ seiching to occur during a given flow case, current results indicated that the transverse and longitudinal waves should have a trough at the downstream inner corner of the cavity simultaneously (scenario named B).

Future research will investigate the relative phase of the different seiching modes more extensively and compare it with other periodic flow features that will be studied using simultaneous velocity measurements. Additionally, the identification of the oscillation states in the experimental recordings will be refined to be more easily applicable for a large number of experiments. It is also planned to investigate more in detail the different superposition scenarios using additional surface measurement devices distributed over the cavity and main channel region. Finally, on-going research is currently investigating the influence of the different seiching modes onto the flow field and mass transport in and around the cavity. This will give new insight in the impact of the different seiching modes onto the flow, and possibly aid better understanding of the intermittent seiching behavior.

\section{ACKNOWLEDGMENTS}

The authors are grateful to the anonymous reviewers for their helpful comments which significantly improved the quality of this paper. L.E. acknowledges support of the Special Research Fund (BOF) of Ghent University as a BOF Fellow. This research was executed with the financial support of the Research Foundation-Flanders (FWO) by the Tournesol Project No. VS.051.18N.

[1] E. Mignot, W. Cai, J. I. Polanco, C. Escauriaza, and N. Rivière, Measurement of mass exchange processes and coefficients in a simplified open-channel lateral cavity connected to a main stream, Environ. Fluid Mech. 17, 429 (2017).

[2] S. Erpicum, T. Meile, B. J. Dewals, M. Pirotton, and A. J. Schleiss, 2D numerical flow modeling in a macro-rough channel, Int. J. Numer. Methods Fluids 61, 1227 (2009).

[3] T. Meile, J.-L. Boillat, and A. Schleiss, Flow resistance caused by large-scale bank roughness in a channel, J. Hydraul. Eng. 137, 1588 (2011). 
[4] C. Juez, I. Bühlmann, G. Maechler, A. J. Schleiss, and M. J. Franca, Transport of suspended sediments under the influence of bank macro-roughness, Earth Surf. Process. 43, 271 (2018).

[5] W. Uijttewaal, D. v. Lehmann, and A. v. Mazijk, Exchange processes between a river and its groyne fields: Model experiments, J. Hydraul. Eng. 127, 928 (2001).

[6] A. McCoy, G. Constantinescu, and L. J. Weber, Numerical investigation of flow hydrodynamics in a channel with a series of groynes, J. Hydraul. Eng. 134, 157 (2008).

[7] V. Weitbrecht, S. A. Socolofsky, and G. H. Jirka, Experiments on mass exchange between groin fields and main stream in rivers, J. Hydraul. Eng. 134, 173 (2008).

[8] A. N. Sukhodolov, Hydrodynamics of groyne fields in a straight river reach: Insight from field experiments, J. Hydraul. Res. 52, 105 (2014).

[9] M. Sanjou and I. Nezu, Fundamental study on mixing layer and horizontal circulation in open-channel flows with rectangular embayment zone, J. Hydrodynam. B 29, 75 (2017).

[10] E. Mignot, W. Cai, G. Launay, N. Rivière, and C. Escauriaza, Coherent turbulent structures at the mixinginterface of a square open-channel lateral cavity, Phys. Fluids 28, 18 (2016).

[11] M. Sanjou and I. Nezu, Hydrodynamic characteristics and related mass-transfer properties in openchannel flows with rectangular embayment zone, Environ. Fluid Mech. 13, 527 (2013).

[12] B. Tuna, E. Tinar, and D. Rockwell, Shallow flow past a cavity: Globally coupled oscillations as a function of depth, Exp. Fluids 54, 1586 (2013).

[13] E. Mignot and W. Brevis, Coherent turbulent structures within open-channel lateral cavities, J. Hydraul. Eng. 146, 8 (2020).

[14] P. Ouro, C. Juez, and M. Franca, Drivers for mass and momentum exchange between the main channel and river bank lateral cavities, Adv. Water Resour. 137, 36 (2020).

[15] B. Tuna and D. Rockwell, Self-sustained oscillations of shallow flow past sequential cavities, J. Fluid Mech. 758, 655 (2014).

[16] Y. Akutina, Experimental Investigation of Flow Structures in a Shallow Embayment Using 3D-PTV, Ph.D. thesis, McGill University, Montreal, QC (2015).

[17] I. Kimura and T. Hosoda, Fundamental properties of flows in open channels with dead zone, J. Hydraul. Eng. 123, 98 (1997).

[18] E. Mignot, M. Pozet, N. Rivière, and S. Chesné, Bidirectional seiching in a rectangular, open channel, lateral cavity, in Proceedings of the 22nd Congrès Français de Mécanique (2015).

[19] R. Tsubaki and I. Fujita, Surface oscillations in flow past a side cavity using stereoscopic measurement and POD, J. Hydrosci. Hydraul. Eng. 24, 41 (2006).

[20] M. Wolfinger, C. Ozen, and D. Rockwell, Shallow flow past a cavity: Coupling with a standing gravity wave, Phys. Fluids 24, 24 (2012).

[21] C. Perrot-Minot, E. Mignot, R. Perkins, D. Lopez, and N. Riviere, Vortex shedding frequency in openchannel lateral cavity, J. Fluid Mech. 892, A25 (2020).

[22] L. Engelen, S. Creëlle, L. Schindfessel, and T. De Mulder, Experimental study of the surface oscillations induced by a shallow flow past a lateral cavity, in Proceedings of the Annual Conference on River Flow 2018, Vol. 40 (EDP Sciences, Les Ulis, France, 2018), p. 05033.

[23] C. Perrot-Minot, L. Engelen, N. Rivière, D. Lopez, T. De Mulder, and E. Mignot, Seiches in lateral cavities with simplified planform geometry: Oscillation modes and synchronization with the vortex shedding, Phys. Fluids 32, 085103 (2020).

[24] C. Torrence and G. P. Compo, A practical guide to wavelet analysis, Bull. Am. Meteor. Soc. 79, 61 (1998).

[25] A. B. Rabinovich, Handbook of Coastal and Ocean Engineering, edited by Y. Kim (World Scientific, Singapore, 2010), pp. 193-236.

[26] C. C. Mei, M. Stiassnie, and D. K.-P. Yue, Theory and Applications of Ocean Surface Waves, Vol. 23 (World Scientific, Singapore, 2005).

[27] O. M. Faltinsen, O. F. Rognebakke, and A. N. Timokha, Resonant three-dimensional nonlinear sloshing in a square-base basin, J. Fluid Mech. 487, 1 (2003).

[28] J. W. Miles, Harbor seiching, Annu. Rev. Fluid Mech. 6, 17 (1974). 
[29] M. O. Freeman, Wavelets signal representations with important advantages, Opt. Photon. News 4, 8 (1993).

[30] A. Teolis and J. J. Benedetto, Computational Signal Processing with Wavelets, Vol. 182 (Springer, Berlin, 1998).

[31] See Supplemental Material at http://link.aps.org/supplemental/10.1103/PhysRevFluids.5.104801 for a video of the surface oscillations inside the cavity during a time window in which the dominant seiching mode switches from transverse to longitudinal seiching. 